BULL. AUSTRAL. MATH. SOC.

VOL. $20(1979), 397-406$.

\title{
A duality theorem for a nondifferentiable nonlinear fractional programming problem
}

\author{
B. Mond and B.D. Craven
}

A duality theorem, and a converse duality theorem, are proved for a nonlinear fractional program, where the numerator of the objective function involves a concave function, not necessarily differentiable, and also the support function of a convex set, and the denominator involves a convex function, and the support function of a convex set. Various known results are deduced as special cases.

Introduction

Let $f: \mathbf{R}^{n} \rightarrow \mathbf{R}, g: \mathbf{R}^{n} \rightarrow \mathbb{R}$, and $h: \mathbb{R}^{n} \rightarrow \mathbb{R}^{m}$ be continuous functions, with $-f$ and $g$ convex. Let $S \subset \mathbb{R}^{m}$ be a closed convex cone, which may in particular be the nonnegative orthant $\mathbf{R}_{+}^{m}$; let the function $h$ be S-convex [6]. Let $c_{1}$ and $c_{2}$ be closed convex sets in $R^{n}$. Consider the nonlinear fractional programing problem

$$
\text { (P): } \operatorname{maximize}_{x \in X_{0}} \frac{f(x)-s\left(x \mid C_{1}\right)}{g(x)+s\left(x \mid C_{2}\right)} \text { subject to }-h(x) \in S \text {, }
$$

in which $x_{0}$ is an open convex set in $\mathbf{R}^{n}, s\left(\cdot \mid c_{i}\right)$ is the support function of the set $C_{i}(i=1,2)$, and it is assumed that

Received 5 April 1979. 


$$
\left.x \in X_{0} \text { (or }-h(x) \in S\right) \Rightarrow g(x)+s\left(x \mid C_{2}\right)>0 \text {. }
$$

Associate to $(P)$ the problem

(D): $\underset{u \in X_{0}, y, z, v, w}{\operatorname{minimize}} z$ subject to $z \geq 0, y \in S^{*}, v \in C_{1}, w \in C_{2}$,

$$
\begin{aligned}
& 0 \in \partial(-f+z g)(u)+\partial\left(y^{T} h\right)(u)+(v+z v), \\
& -f(u)+z g(u)+y^{T} h(u)+(v+z w)^{T} u \geq 0 .
\end{aligned}
$$

In (D), $S^{*}$ is the dual cone to $S$ [6], and $\partial$ denotes subdifferential [15].

Under suitable hypothesis, (D) will be shown to be a dual problem to (P); under somewhat different assumptions, (P) will be shown to be a dual problem to $(D)$.

The constraint $-h(x) \in S$ is locally solvable [4], [5] at $x_{0}$ if $-h\left(x_{0}\right) \in S$ and, for some $\delta>0$, whenever the direction $d$ satisfies $-h\left(x_{0}\right)-h^{\prime}\left(x_{0} ; d\right) \in S$ and $\|d\|<\delta \quad$ (where $h^{\prime}\left(x_{0} ; d\right)$ denotes directional derivative in direction $d$ ), there exists a solution $x=x_{0}+\alpha d+o(\alpha)$ to $-h(x) \in S$, valid for sufficiently small $\alpha>0$. (This requirement reduces to the Kuhn-Tucker constraint qualification for a constraint system $\left.h_{i}(x) \leq 0 \quad(i=1,2, \ldots, m).\right)$ The problem (P) will be said to satisfy a constraint qualification at $x_{0}$ if $-h\left(x_{0}\right) \in S$, and either

(a) Slater's constraint qualification holds, namely $-h(x) \in$ int $S$ for some $x \in X_{0}$, or

(b) $-h(x) \in S$ is locally solvable at $x_{0} \in X_{0}$, and the set

$$
\underset{s \in S^{*}}{U}\left\{\operatorname{sh}\left(x_{0}\right)\right\} \times \partial(\operatorname{sh})\left(x_{0}\right)
$$$$
\text { is closed in } \mathbb{R} \times \mathbf{R}^{m} \text {. }
$$

(The latter is automatic if $S$ is a polyhedral cone and $h$ is differentiable at $x_{0}$ [9].) 


\section{Duality theorem}

The assumptions stated in the Introduction will be assumed throughout.

THEOREM 1. Weak duality holds for (P) and (D), namely $\sup (P) \leq \inf (D)$. If $(P)$ reaches a maximum at $x=x_{0} \in X_{0}$, if $\max (P) \geq 0$, and if a constraint qualification holds for (P), then (D) reaches a minimum at some $(u, z, y, v, w)$ with $u=x_{0}$, and $\max (P)=\min (D)$.

Thus (D) is a strong dual [7], [8] to (P).

Proof. Let $x$ be feasible for (P), and let $(u, z, y, v, w)$ be feasible for (D). From a constraint for (D), $\theta+\psi+(v+z w)=0$ for some $\theta \in \partial \varphi(u)$ and some $\psi \in \partial\left(y^{T} h\right)(u)$, where $\varphi=-f+z g$. Since $z \geq 0$ and $-f$ and $g$ are convex, $\varphi$ is convex. Then

$$
\begin{aligned}
{\left[f(x)-s\left(x \mid C_{1}\right)\right] } & -z\left[g(x)+s\left(x \mid C_{2}\right)\right] \\
& =f(x)-z g(x)-(v+z w)^{T} x \\
& =-\varphi(x)-(v+z \omega)^{T} x \\
& \leq-\varphi(u)-\theta^{T}(x-u)-(v+z w)^{T}(x-u)-(v+z w)^{T} u \text { since } \theta \in \partial \varphi(u) \\
& =-\varphi(u)-(v+z \omega)^{T} u+\psi^{T}(x-u) \text { by a constraint for (D) } \\
& \leq y^{T} h(u)+\psi^{T}(x-u) \text { by a constraint for (D) } \\
& \leq y^{T} h(x) \text { since } y^{T} h \text { is a convex function } \\
& \leq 0 \text { since }-h(x) \in S \text { and } y \in S^{*} \text {. }
\end{aligned}
$$

By hypothesis, $g(x)+s\left(x \mid C_{2}\right)>0$. Dividing by it,

$$
\left[f(x)-s\left(x \mid C_{1}\right)\right] /\left[g(x)+s\left(x \mid C_{2}\right)\right] \leq z
$$

Hence $\sup (P) \leq \inf (D)$.

I.ow assume that $(P)$ is maximized at $x_{0}$, with $m=\max (P) \geq 0$, and also the constraint qualification. Then $x_{0}$ also maximizes

$$
\left[f(x)-s\left(x \mid C_{1}\right)\right]-m\left[g(x)+s\left(x \mid C_{2}\right)\right]
$$

subject to $-h(x) \in S$. Applying the appropriate nondifferentiable version of the Kuhn-Tucker Theorem, assuming constraint qualification (a) or (b) 
(see [9], Theorem 4),

$$
0 \in \partial(-f+m g)\left(x_{0}\right)+v+w+\partial\left(y^{T} h\right)\left(x_{0}\right), \quad y^{T} h\left(x_{0}\right)=0,
$$

holds for some $y \in S^{*}, v \in \partial s\left(x_{0} \mid C_{1}\right)$, and $w \in \partial s\left(x_{0} \mid C_{2}\right)$. Then [15], $\imath \in C_{1}, v^{T} x_{0}=s\left(x_{0} \mid C_{1}\right), w \in C_{2}, w^{T} x_{0}=s\left(x_{0} \mid C_{2}\right) ;$ therefore all constraints of (D) except (4) hold for $u=x_{0}, z=m, y, v, w$, and (4) holds also from $\left[f\left(x_{0}\right)-s\left(x_{0} \mid C_{1}\right)\right] /\left[g\left(x_{0}\right)+s\left(x_{0} \mid C_{2}\right)\right]=m$ and $y^{T} h\left(x_{0}\right)=0 . \square$

\section{Converse duality theorem}

Again assume all assumptions of the Introduction, including (2). Denote by $F(x)$ the objective function for $(\mathrm{P})$.

Suppose that (3) and (4) are satisfied for

$$
(u, z, y, v, w)=\left(u_{0}, z_{0}, y_{0}, v_{0}, w_{0}\right),
$$

where $y_{0} \in S^{*}, v_{0} \in C_{1}, w_{0} \in C_{2}$, and $g\left(u_{0}\right)+w_{0}^{T} u_{0}>0$. The system

$$
0 \in \partial(-f+z g)(u)+\partial\left(y^{T} h\right)(u)+(v+z w),
$$

together with

$$
z=\left[f(u)-v^{T} u-y^{T} h(u)\right] /\left[g(u)+w^{T} u\right],
$$

will be called solvable near $u_{0}$ if, whenever $y=y_{0}+\beta \tilde{y} \in S^{*}$, $v=v_{0}+\beta \tilde{v} \in C_{1}$, and $w=w_{0}+\beta \tilde{w} \in C_{2}$, for $0 \leq \beta \leq I$, then the system (3) and (7) has a solution $u=u_{0}+\tilde{u}(\beta)$ for all sufficiently small positive $\beta$, satisfying $\tilde{u}(\beta) \rightarrow 0$ as $\beta \downarrow 0$.

This property holds, in particular, if $-f+z_{0} g+y_{0}^{T}$ has a nonsingular hessian matrix, at $u=u_{0}$, in consequence of the implicit function theorem. However, the following converse duality theorem does not assume any differentiability of the functions $f, g, h$.

THEOREM 2. Let (D) reach a minimum at

$$
(u, z, y, v, w)=\left(u_{0}, z_{0}, y_{0}, v_{0}, w_{0}\right),
$$


where $u_{0} \in X_{0}$. If $z_{0}=0$, assume that $F(\hat{x}) \geq 0$ for some $\hat{x} \in X_{0}$ satisfying $-h(\hat{x}) \in S$. If $z_{0}>0$, assume that the system (3) and (7) is solvable near $u_{0}$. Then $(\mathrm{P})$ reaches a maximum, and $\max (\mathrm{P})=\min (\mathrm{D})$. Hence $(P)$ is a strong dual to (D).

Proof. If $z_{0}=0$, then $F(\hat{x}) \leq z_{0}=0$ by weak duality, also $F(\hat{x}) \geq 0$ by assumption. Hence $F(\hat{x})=z_{0}$, and weak duality implies that $\hat{x}$ is optimal for $(P)$, so that $\max (P)=\min (D)$. (Weak duality is available from Theorem 1.)

Suppose now that $z_{0} \neq 0$; since $z_{0} \geq 0$ for $(D), z_{0}>0$. Choose any $\tilde{y}, \tilde{v}, \tilde{w}$ so that $y_{0}+\tilde{y} \in S^{*}, v_{0}+\tilde{v} \in C_{1}, w_{0}+\tilde{w} \in C_{2}$. Since $S^{*}, C_{1}$, and $C_{2}$ are convex sets, $y=y_{0}+\beta \tilde{y} \epsilon S^{*}, v=v_{0}+\beta \tilde{v} \epsilon C_{1}$, and $w=w_{0}+\beta \tilde{w} \in C_{2}$, whenever $0 \leq \beta \leq 1$. By assumption $g\left(u_{0}\right)+w_{0}^{T} u_{0}>0$, and then (3) and (7) have a solution $u=u_{0}+\tilde{u}(\beta)$ for sufficiently small $\beta>0$. By continuity, $g(u)+w^{T} u>0$ for sufficiently small $\beta>0$; hence (7) implies (4). Hence this point $(u, z, y, v, w)$, with $u=u_{0}+\tilde{u}(\beta)$ and $z$ given by $(7)$, is feasible for (D), for sufficiently small $\beta>0$.

Since $\left(u_{0}, z_{0}, y_{0}, v_{0}, w_{0}\right)$ minimizes (D),

$$
z_{0} \leq\left[f(u)-v^{T} u-y^{T} h(u)\right] /\left[g(u)+w^{T} u\right] \equiv p / q,
$$

using (7), where

$$
\begin{array}{r}
p=f\left(u_{0}\right)+\beta f^{\prime}\left(u_{0} ; \tilde{u}\right)-v_{0}^{T} u_{0}-\beta v_{0}^{T} \tilde{u}-\beta \tilde{v}^{T} u_{0}-y_{0}^{T} h\left(u_{0}\right) \\
\quad-\beta y_{0}^{T} h^{\prime}\left(u_{0} ; \tilde{u}\right)-\beta \tilde{y}^{T} h\left(u_{0}\right)+o(\beta),
\end{array}
$$$$
\text { (10) } q=g\left(u_{0}\right)+\beta g^{\prime}\left(u_{0} ; \tilde{u}\right)+w_{0}^{T} u_{0}+\beta w_{0}^{T} \tilde{u}+\beta \tilde{w}^{T} u_{0}+o(\beta) \text {. }
$$

Combining these terms shows that

$$
\left(p_{0}-z_{0} q_{0}\right)+\beta R-\beta\left[\tilde{y}^{T} h\left(u_{0}\right)+\left(\tilde{v}+z_{0} \tilde{w}\right)^{T} u_{0}\right]+o(\beta) \geq 0,
$$


where $p_{0}=f\left(u_{0}\right)+y_{0}^{T} h\left(u_{0}\right)+v_{0}^{T} u_{0}, q_{0}=g\left(u_{0}\right)+w_{0}^{T} u_{0}$, and

$$
R=f^{\prime}\left(u_{0} ; \tilde{u}\right)-z_{0} g^{\prime}\left(u_{0} ; \tilde{u}\right)-\left(y_{0}^{T} h\right)^{\prime}\left(u_{0} ; \tilde{u}\right)-v_{0}^{T} \tilde{u}-z_{0} w_{0}^{T} \tilde{u} \text {. }
$$

Then $p_{0}-z_{0} q_{0} \geq 0$; but $p_{0}-z_{0} q_{0} \leq 0$ by (4), so $p_{0}-z_{0} q_{0}=0$. Dividing (11) by $\beta$ and letting $\beta+0$, then shows that

$$
R-\tilde{y}^{T} h\left(u_{0}\right)-\left(\tilde{v}+z_{0} \tilde{w}\right)^{T} u_{0} \geq 0 \text {. }
$$

Denote $\psi=-f+z_{0} g+y^{T} h$. From (3), $\theta+v_{0}+z_{0} w_{0}=0$ for some $\theta \in \partial \psi\left(u_{0}\right)$. Then $\psi^{\prime}\left(u_{0} ; \tilde{u}\right) \geq \theta^{T} \tilde{u}$. Since $R=\dot{\psi}^{\prime}\left(u_{0} ; \tilde{u}\right)-\left(v_{0}+z_{0} w_{0}\right)^{T} \tilde{u}$, it follows that $R \leq-\left(\theta+v_{0}+z w_{0}\right)^{T} \tilde{u}=0$. Hence

$$
\tilde{y}^{T} h\left(u_{0}\right)+\left(\tilde{v}+z_{0} \tilde{w}\right)^{T} u_{0} \leq 0 \text {. }
$$

Setting $\tilde{y}=0$ and $\tilde{w}=0, \tilde{v}^{T} u_{0} \leq 0$ whenever $v_{0}+\tilde{v} \epsilon C_{1}$. Hence $v^{T} u_{0} \leq v_{0}^{T} u_{0}$ for each $v \in C_{1}$. Therefore $s\left(u_{0} \mid C_{1}\right) \leq v_{0}^{T} u_{0}$. Since $v_{0} \in C_{1}$, by a constraint of $(D), s\left(u_{0} \mid C_{1}\right) \geq v_{0}^{T} u_{0}$. Hence $v_{0}^{T} u_{0}=s\left(u_{0} \mid C_{1}\right)$. Since $z_{0}>0$, a similar argument applied to $\tilde{w}^{T} u_{0}$ shows that $w_{0}^{T} u_{0}=s\left(u_{0} \mid C_{2}\right)$. Now let $\tilde{v}=0$ and $\tilde{w}=0$. Then $\tilde{y}^{T} h\left(u_{0}\right) \leq 0$ whenever $\tilde{y} \in S^{*} ;$ since $S$ is a closed convex cone, it follows that $-h\left(u_{0}\right) \in S$. Setting $\tilde{y}=-3_{2} y_{0}, y_{0}+\tilde{y} \in S^{*}$, and then $\left(-b_{0}\right)^{T} h\left(u_{0}\right) \leq 0$. But also $y_{0}^{T} h\left(u_{0}\right) \leq 0$ since $y_{0} \in S^{*}$, and $-h\left(u_{0}\right) \in S$ has just been proved. Therefore $y_{0}^{T} h\left(u_{0}\right)=0$.

Thus $u_{0}$ is feasible for $(P)$, and the optimal objective function for (D) equals

$$
z_{0}=\left[f\left(u_{0}\right)-s\left(u_{0} \mid C_{1}\right)-0\right] /\left[g\left(u_{0}\right)+s\left(u_{0} \mid C_{2}\right)\right]=F\left(u_{0}\right) .
$$

Using weak duality, it follows that $u_{0}$ is optimal for (P). Hence 
$\max (P)=\min (D)$.

\section{Discussion and examples}

If $f, g$, and $h$ are differentiable functions, then (3) reduces to

$$
0=\left(-f+z g+y^{T} h\right) '(u)+(v+z w) \text {. }
$$

For nondifferentiable functions, an equivalent to (3) is (see [15])

$$
\text { (for all } t) \varphi^{\prime}(u ; t)+(v+z \omega) t \geq 0,
$$

where $\varphi=-f+z g+y^{T} h$.

The technique of proof for Theorem 2 is adapted from that of [9], Theorem 6, and [5], Theorem 4.8.1. Since the "solvable near $u_{0}$ "requirement in Theorem 2 does not demand a unique solution for $u$, the usual implicit function theorem is assuming too much. A general verifiable solvability criterion for the convex nondifferentiable case has yet to be found. An inclusion of the form $\rho \in \partial \Phi(u)$ must be solved (nonuniquely) for $u$, given $\rho_{0} \in \partial \Phi\left(u_{0}\right)$. For this it suffices if $\partial \Phi(\cdot)$ maps a neighbourhood of $u_{0}$ onto some neighbourhood of $\rho_{0}$. As a simple example let $\Phi(u)=\left(u^{T} u\right)^{\frac{3}{2}}+\frac{1}{3} \varepsilon u^{T} u$, for $u \in \mathbf{R}^{n}$ and $\varepsilon$ a positive constant; set $u_{0}=0$. Denote $B(r)=\left\{\xi \in \mathbf{R}^{n}:\|\xi\| \leq r\right\}$. Then $\partial \Phi\left(u_{0}\right)=B(1)$, and $\partial \Phi(\cdot)$ maps $B(\delta)$ onto $B(1+\varepsilon \delta)$, so that the sufficient requirement is fulfilled, provided that $\left\|\rho_{0}\right\| \leq 1$, for this nondifferentiable function $\Phi$. This is not so if $\varepsilon=0$.

Problem (P) includes various special cases. If $f, g$, and $h$ are affine, then (P) reduces to that considered by Mond and Schechter [13], [14]. As noted in [13], if $B$ is a positive semidefinite matrix, and $Q$ is the compact set $\left\{B v: v^{T} B v \leq 1\right\}$, then

$$
s(x \mid Q)=\left(x^{T} B x\right)^{\frac{1}{2}} \text {. }
$$

Thus, if $f, g$, and $h$ are differentiable functions, and $s\left(\cdot \mid C_{1}\right)$ and $s\left(\cdot \mid c_{2}\right)$ are defined as in (18) by positive semidefinite matrices $B$ and $D$, problem $(P)$ becomes the nondifferentiable fractional programming 
problem considered in [11]. If also $f, g$, and $h$ are affine, the results of Chandra and Gulati [3] are obtained. If $g(x) \equiv 1$, and $C_{2}=\{0\}$, then $s\left(x \mid C_{2}\right)=0$, so that a nonfractional nondifferentiable objective function $F(x)=f(x)-s\left(x \mid C_{1}\right)$ is recovered.

If $S$ is a $k \times n$ matrix, then [13]

$$
\|S x\|_{p}=s(x \mid Q)
$$

for $p \geq 1$, where $Q=\left\{S^{T} u:\|u\|_{q} \leq 1\right\}$, and $p^{-1}+q^{-1}=1$, and $q=\infty$ if $p=1$. Here $\|x\|_{p}=\left[\sum\left|x_{i}\right|^{p}\right]^{1 / p}$ if $p<\infty$, and $\|x\|_{\infty}=\sup \left\{\left|x_{i}\right|: i=1,2, \ldots\right\}$. The set $Q$, as defined, is convex and compact.

Thus if $f, g$, and $h$ are differentiable, $s\left(\cdot \mid C_{1}\right)$ is defined as in (19) by a matrix $S_{1}$ and a scalar $p_{1}$, and similarly $s\left(\cdot \mid C_{2}\right)$ by a matrix $S_{2}$ and a scalar $p_{2}$, then problems $(P)$ and $(D)$ become respectively

$\left(P^{\prime}\right): \operatorname{maximize}_{x \in X}\left[f(x)-\left\|S_{1} x\right\|_{p_{1}}\right] /\left[g(x)+\left\|S_{2} x\right\|_{p_{2}}\right]$ subject to $h(x) \leq 0$,

(D'): $\underset{u \in X_{0}, z, y, v, w}{\operatorname{minimize}} z$ subject to $z \geq 0, y \geq 0,\|v\|_{q_{1}} \leq 1,\|w\|_{q_{2}} \leq 1$,

$$
\begin{gathered}
\nabla\left(y^{T} h-f+z g\right)(u)+S_{1}^{T} v+S_{2}^{T} w=0 \\
-f(u)+z g(u)+y^{T} h(u)+u^{T}\left(S_{1}^{T} v+S_{2}^{T} w\right) \geq 0 .
\end{gathered}
$$

If, in particular, $f, g$, and $h$ are affine functions, then some of the problems discussed in [13] are obtained.

If $f, g$, and $h$ are differentiable, and $C_{2}$ consists only of the zero vector in $\mathbb{R}^{n}$, and $s\left(\cdot \mid C_{1}\right)$ is defined, as in (18), by a positive semidefinite matrix $B$, then $(P)$ and $(D)$ reduce to the problems considered by Aggarwal and Saxena [1], [2]. If also $g(x) \equiv 1$, one obtains the (nonfractional) problems discussed in $[10]$. 
If $f, g$, and $h$ are differentiable, $C_{2}=\{0\}, g(x) \equiv 1$, and $s\left(\cdot \mid C_{1}\right)$ is defined as in (19) by a matrix $S$, then the present results yield those of Mond and Schechter [12].

\section{References}

[1] S.P. Aggarwal, P.C. Saxena, "Duality theorems for non-linear fractional programs", 2. Angew. Math. Mech. 55 (1975), 523-525.

[2] S.P.'Aggarwal \& P.C. Saxena, "A class of fractional functional programming problems", New Zealand Oper. Res. 7 (1979), 79-90.

[3] Suresh Chandra and T.R. Gulati, "A duality theorem for a nondifferentiable fractional programming problem", Management Sci. $23(1976 / 77), 32-37$.

[4] B.D. Craven, "Lagrangean conditions and quasiduality", BulZ. Austral. Math. Soc. 16 (1977), 325-339.

[5] B.D. Craven, Mathematical programming and control theory (Chapman and Hall, London; John Wiley \& Sons, New York; 1978).

[6] B.D. Craven and B. Mond, "Transposition theorems for cone-convex functions", SIAM J. Appl. Math. 24 (1973), 603-612.

[7] B.D. Craven and B. Mond, "The dual of a fractional linear program", J. Math. Anal. Appl. 42 (1973), 507-512.

[8] B.D. Craven and B. Mond, "The dual of a fractional linear program: Erratum", J. Math. Anal. Appl. 55 (1976), 807.

[9] B.D. Craven and B. Mond, "Lagrangean conditions for quasidifferentiable optimization", Survey of mathematical programing $I$ (Proc. Ninth Intern. Sympos. Mathematical Programming, Budapest, 1976. Iorth-Holland, to appear).

[10] Bertram iond, "A class of nondifferentiable mathematical programming problems", J. irath. Anal. Appl. 46 (1974), 169-174.

[11] B. Mond, "A class of nondifferentiable fractional programming problems", 2. Angew. Wath. Wech. 58 (1978), 337-341. 
[12] Bertram Mond and Murray Schechter, "A programming problem wi.th an $L_{p}$ norm in the objective function", J. Austral. Math. Soc. Ser. B $19(1975 / 1976), 333-342$.

[13] B. Mond and M. Schechter, "A duality theorem for a homogeneous fractional programming problem", J. Optim. Theory Appl. 25 $(1978), 349-359$.

[14] B. Mond and M. Schechter, "Converse duality in homogeneous fractional programming", preprint, 1979.

[15] R. Tyrrell Rockafellar, Convex analysis (Princeton University Press, Princeton, New Jersey, 1970).

Department of Pure Mathematics,

La Trobe University,

Bundoora,

victoria;

Department of Mathematics,

University of Melbourne,

Parkville,

victoria. 